\title{
Review
}

\section{New targets for the antitumor activity of gambogic acid in hematologic malignancies}

\author{
Li-jing YANG, Yan CHEN* \\ Department of Hematology, Union Hospital, Tongji Medical College, Huazhong University of Science and Technology, Wuhan \\ 430022, China
}

\begin{abstract}
Gambogic acid (GA) is the main active ingredient of gamboge, a brownish to orange dry resin secreted from Garcinia hanburyi, a plant that is widely distributed in nature. Recent in vitro and in vivo studies have demonstrated that GA exerts potent antitumor effects against solid tumors of various derivations, and its antitumor mechanisms have been thoroughly investigated. On the other hand, normal cells remain relatively resistant to GA, indicating a therapeutic window. GA is currently in clinical trials in China. Over the last decade, our laboratory demonstrates that GA exhibits potent anticancer activities against hematological malignancies. This review focuses on the new mechanisms through which GA inhibits proliferation and induces apoptosis in malignant hematological cells. These include the regulation of expression and intracellular positioning of nucleoporin and nucleophosmin; downregulation of steroid receptor coactivator-3 (SRC-3) and its downstream proteins; upregulation of death inducer-obliterator (DIO-1); downregulation of HERG potassium channel; as well as induction of reactive oxygen species (ROS) accumulation.
\end{abstract}

Keywords: gambogic acid; hematological malignancies; death inducer-obliterator; HERG channel; nucleophosmin; nucleoporin; steroid receptor coactivator-3; reactive oxygen species

Acta Pharmacologica Sinica (2013) 34: 191-198; doi: 10.1038/aps.2012.163; published online 31 Dec 2012

\section{Introduction}

It is extraordinarily important to maintain a balance between cell division and death to ensure the correct development and maintenance of multicellular organisms. Disruption of this dynamic balance has pathologic consequences and can lead to disturbed embryogenesis, neurodegenerative diseases, and tumor formation ${ }^{[1]}$. The multi-step process of tumor generation is typically linked to apoptosis resistance. For example, by interrupting the signals that can trigger mitochondrial perturbations, tumor cells gain the ability to abolish the mitochondrial pathway of apoptosis ${ }^{[2]}$. Additionally, the inability of most cancers to undergo apoptosis in response to chemotherapeutics is the key cause of treatment failure and presents one of the major unsolved bottlenecks in oncology $y^{[3]}$.

In recent decades, natural drugs have received much attention as cancer chemopreventive agents and therapeutics. Because of their capacity to bind multiple targets, natural drugs may have an advantage over rationally designed monotargeted agents in the treatment of cancer with multigenic abnormalities $^{[4]}$. The use of herbs in China can be dated back

\footnotetext{
* To whom correspondence should be addressed.

E-mail yanchen@public.wh.hb.cn

Received 2012-08-14 Accepted 2012-11-02
}

to the third century BCE, and more than 7000 species have been recorded in Chinese literature since then. One of these species, the gamboges, has been reported to cure tooth decay and edema in ancient Chinese books.

Gambogic acid (GA, $\mathrm{C}_{38} \mathrm{H}_{44} \mathrm{O}_{8}, \mathrm{MW}$ : 628.75, Figure 1) is the main active ingredient in gamboges and is a brownish to orange dry resin secreted from Garcinia hanburyi, a plant that mainly grows in South China, Cambodia, Vietnam, and Thailand ${ }^{[5]}$. Recent in vitro and in vivo studies demonstrated that GA had potent anti-tumor effects on solid tumors of various derivations, such as human lung carcinoma ${ }^{[6]}$ and hepatoma ${ }^{[7]}$. The mechanisms of GA anti-tumor activity include downregulating Bcl-2, activating p53, directly bind-

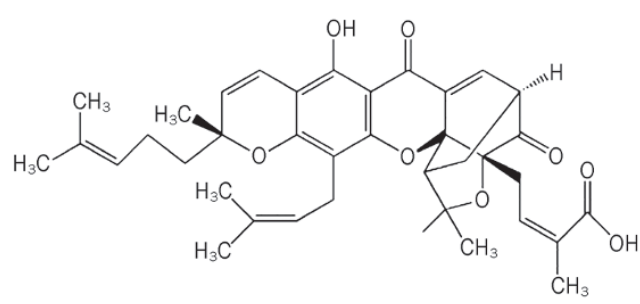

Figure 1. Chemical structure of Gambogic acid (GA, $\mathrm{C}_{38} \mathrm{H}_{44} \mathrm{O}_{8}, \mathrm{MW}$ : 628.75). 
ing to c-myc and transferring receptors, and blocking vascular endothelial growth factor (VEGF) signaling ${ }^{[4]}$. Additionally, GA3, a new GA derivative, can produce potent cytotoxicity against a panel of cell lines ${ }^{[8]}$. In contrast, GA exhibits less toxicity to normal cells compared with cancer cells. For example, normal hepatocytes and breast cells are more resistant to GA than hepatoma and breast cancer cells ${ }^{[4,9]}$, indicating a therapeutic window. Over the last decade, our laboratory has demonstrated that GA exerts potent anticancer activity in hematological malignancies. This review will focus on the mechanisms through which GA kills malignant hematological tumor cells (Figure 2).

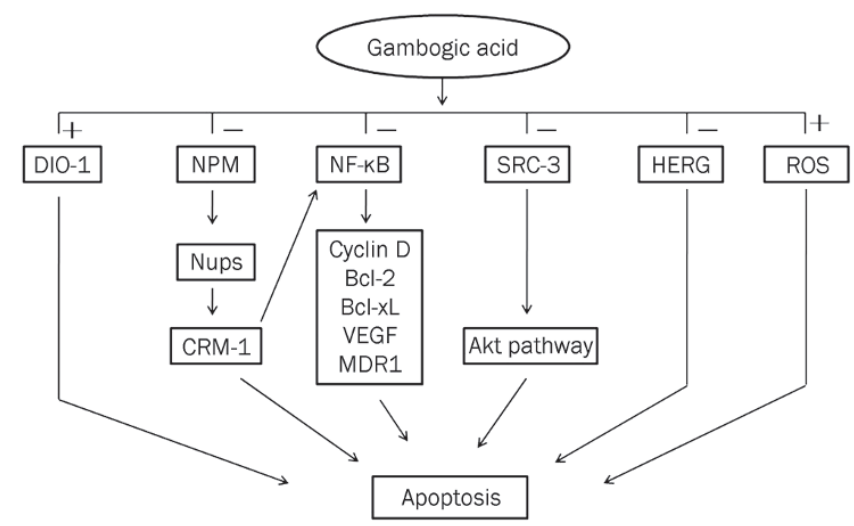

Figure 2. The anti-hematologic malignancies mechanisms of GA. GA induces hematologic malignancies cells apoptosis via regulating the expression and intracellular positioning of nucleoporin and nucleophosmin (NPM); down-regulating steroid receptor coactivator-3 (SRC-3) as well as its downstream proteins; up-regulating death inducer-obliterator (DIO-1); down-regulating NF-kB; down-regulating HERG potassium channel; and induction of ROS accumulation.

\section{Regulation of nucleoporin and nucleophosmin}

Because various proteins are abnormally localized in the cytoplasm and nucleus of tumor cells, mechanisms relating to the nucleocytoplasmic transport of proteins may provide novel opportunities for anti-cancer drug research ${ }^{[10]}$. As the only nucleocytoplasmic pore, the nuclear pore complex (NPC) spans the two lipid bilayers of the nuclear envelope and is an essential mediator of all known transport events between the nucleus and the cytoplasm. In addition to mediating nucleocytoplasmic transport, the NPC seems to be either directly or indirectly involved in many other cellular processes, including chromosome segregation, gene expression and apoptosis. The NPC consists of more than 30 different proteins, named nucleoporins (Nups) ${ }^{[11]}$.

Nup88, an NPC protein, has received considerable attention as a potential marker for high-grade tumors. Nup88 is a nonphenylalanine-glycine (FG) nucleoporin located exclusively on the cytoplasmic side of the $\mathrm{NPC}^{[12]}$. It contains two repeating structural motifs: the N-terminal domain, which is predicted to form a $\beta$-propeller structure, and the C-terminal domain, which is predicted to contain coiled-coils ${ }^{[13]}$. Nup88 can form a sub-complex of the NPC together with the FG repeat nucleoporin Nup214 through the N-terminal $\beta$-propeller structure of Nup88 and a central coiled-coil domain of Nup214 $4^{[12]}$. This Nup88/Nup214 sub-complex makes important contributions to nuclear export. It has been reported that both the FG repeat domain of Nup214 and the N-terminal $\beta$-propeller domain can bind directly to CRM-1/exportin- $1^{[14,15]}$, the receptor for the export of most proteins from the nucleus, such as nuclear factor kappaB (NF-kB ${ }^{[16]}$, which is a ubiquitous transcription factor involved in the immune response, apoptosis, and cancer.

A monoclonal antibody raised against Candida albicans unexpectedly cross-reacted with human Nup8 ${ }^{[17]}$, leading to the discovery of Nup88 overexpression in a broad spectrum of neoplasias, including carcinomas, sarcomas, lymphomas and mesotheliomas ${ }^{[18]}$. Immunoblotting several lung carcinoma samples demonstrated that Nup88 expression did not correlate with a concomitant overexpression of its interacting partner Nup214, suggesting selective nucleoporin dysregulation ${ }^{[18]}$. Moreover, the intensity of Nup88 staining generally correlates with tumor grade, and the highest expression is routinely detected in more advanced tumors and around the edges of tumors, suggesting a link to invasivity ${ }^{[19]}$.

Until now, the exact nature of the relationship between Nup88 overexpression and tumorigenesis has been uncertain. Perhaps, the overexpression of Nup88 leads to the assembly of the Nup88/Nup214 subcomplex, which traps CRM-1 at cytoplasmic foci and inhibits protein export ${ }^{[15]}$. For example, the depletion of Nup88 by small interfering RNA (siRNA) inhibited NF-kB-dependent reporter gene activation and the nuclear translocation of NF- $\mathrm{kB}$ without affecting the upstream activation pathway in mouse NIH3T3 fibroblast cells. In metastatic melanoma cells overexpressing Nup88, constitutive NF-kB activation was found in the nucleus and cytoplasm, and Nup88 depletion reduced the tumor necrosis factor (TNF)-induced nuclear accumulation of NF-KB subunits in these cells. These phenomena imply that Nup88 overexpression in tumor cells may contribute to constitutive NF-kB activation ${ }^{[20]}$.

In our previous study, almost $96 \%$ of acute monocytic leukemia U937 cells expressed Nup88 by flow cytometry analysis. It has been reported that Nup88 is normally localized on the cytoplasmic side of NPCs ${ }^{[12]}$, but in U937 cells, immunofluorescence detection using confocal microscopy showed that it is extensively expressed in the nucleus and cytoplasm ${ }^{[21]}$. Compared with the control group, Nup88 in U937 cells treated with $1.0 \mathrm{mg} / \mathrm{L} \mathrm{GA}$ for $24 \mathrm{~h}$ was re-distributed to the cytoplasmic side of NPCs. GA could correct the disrupted distribution of Nup88 in U937 cells and decrease the protein level by inhibiting gene transcription in a dose-dependent manner. As a result of the effect of GA on the regulation of Nup88, the function of nucleocytoplasmic traffic was affected, leading to the failure to meet the great demand for proteins in U937 cells. GA also downregulated the expression of Nup88 protein and mRNA levels in human HL-60 myeloid leukemia cells ${ }^{[22]}$ and Jurkat $\mathrm{T}$ cell leukemia cells ${ }^{[23]}$, which was accompanied by cell apoptosis. 
Nucleophosmin is another key molecule in nucleocytoplasmic communication. It can shuttle continuously through the NPC between the nucleus and the cytoplasm during the cell cycle and sustains normal cell homeostasis ${ }^{[24]}$. The nucleophosmin gene (nucleophosmin1) belongs to a new category of genes that function as oncogenes or tumor-suppressor genes depending on its dosage, expression level, interacting partners and compartmentalization ${ }^{[25]}$. It has been reported that the nucleophosmin 1 gene is one of the most frequent targets of genetic alterations in hematological malignancies, especially in de novo acute myeloid leukemia ${ }^{[26]}$. In pharmaceutical research on tumor treatments, the change in nucleophosmin expression is relevant to the destiny of cancer cells. For example, nucleophosmin expression is decreased in phorbol ester 12-otetradecanoylphorbol-13-acetate (TPA)-induced human K562 chronic myeloid leukemia cell differentiation ${ }^{[27]}$ and retinoic acid-induced differentiation and sodium butyrateinduced apoptosis of HL-60 cells ${ }^{[28]}$. In our previous research, GA decreased the expression of Nup88 and changed its location and decreased the expression of nucleophosmin in a dosedependent manner ${ }^{[23]}$.

Altogether, these results demonstrated that GA has a potent ability to block the nucleocytoplasmic transport of proteins by regulating Nup88 and nucleophosmin, which results in apoptosis and the cell cycle arrest of Jurkat cells, HL60 cells and U937 cells.

\section{Regulation of steroid receptor coactivator-3 (SRC-3)}

SRC-3 is a member of the p160 family of SRCs, which also contains SRC-1 and SRC-2/TIF-2/GRIP-1 ${ }^{[29]}$. SRC-3 was first identified from an amplified region of the long arm of chromosome 20 (20q) in breast cancer in 1997 by Anzick SL and originally termed amplified in breast cancer 1 (AIB1) ${ }^{[30]}$. SRC-3 also has other names, such as nuclear receptor coactivator-3 (NCoA-3), receptor-associated coactivator-3 (RAC3), activator of thyroid hormone and retinoid receptor (ACTR), thyroid hormone receptor-activating molecule-1 (TRAMI), and $\mathrm{p} 300 / \mathrm{CBP}$-interacting protein $(\mathrm{p} / \mathrm{CIP})^{[31]}$. The SRC-3 protein contains three basic structural domains: the N-terminal basic helix-loop-helix-Per/ARNT/Sim domain (bHLH-PAS), through which SRC-3 interacts with other DNA-binding proteins; the receptor-interacting domain (RID), which includes three LXXLL motifs and through which SRC-3 binds to the ligand-activated nuclear receptors; and the C-terminal domain, which contains two intrinsic transcriptional activation domains named AD1 and AD2 and contributes to the interaction between SRC-3 and histone acetyltransferases or methyltransferases. The C-terminal of SRC-3 also has its own histone acetyltransferase activity ${ }^{[32]}$.

Since SRC-3 was first discovered to be amplified in breast tumors in 1997, the correlation between SRC-3 and tumors has been comprehensively discussed ${ }^{[33]}$. Recently, a growing body of evidence has demonstrated that SRC-3 plays a central role in tumor genesis, progression, invasion and metastasis. SRC-3 is often overexpressed in several hormone-dependent cancers, such as breast cancer ${ }^{[33]}$, ovarian cancer ${ }^{[34]}$, and endo- metrial cancer ${ }^{[35]}$. Moreover, SRC-3 dysregulation also leads to hormone-independent cancers and malignant disease, such as colorectal cancer ${ }^{[36]}$, hepatocellular cancer $(\mathrm{HCC})^{[37]}$, pancreatic cancer $^{[38]}$, and gastric cancer ${ }^{[39]}$. In animal models, overexpression of SRC-3 results in increasing cancer incidence in various organs including the lung, uterus, and breast $\mathrm{t}^{[40]}$. With regard to hematological malignancies, SRC-3 has been found to be overexpressed in K562 chronic myelogenous leukemia cells and Raji Burkitt's lymphoma cells ${ }^{[4]}$. Additionally, SRC-3 is involved in leukemia-specific chromosome translocation in acute myelocytic leukemia (AML)-M4/M5, which leads to the expression of the MYST3/NCOA3 fusion gene ${ }^{[42]}$.

SRC-3 is related to the apoptosis resistance of cancer cells. The overexpression of SRC-3 partly blocked $\mathrm{H}_{2} \mathrm{O}_{2}$-mediated apoptosis in HEK293 cells in vitro ${ }^{[43]}$. Overexpression of SRC-3 in $\mathrm{K} 562$ cells has been implicated in cancer multidrug resistance, as the inhibition of SRC-3 by siRNA enhanced the sensitivity of K562 cells to TNF-related apoptosis-inducing ligand (TRAIL)-mediated apoptosis ${ }^{[4]}$. These results implied that SRC-3 had the potential to be a new target for antitumor drug research. Additionally, we confirmed that GA could downregulate the levels of both SRC-3 mRNA and protein in A549 cells and K562 cells ${ }^{[44]}$. It has been reported that SRC-3 can disturb apoptosis through Akt signaling, which is related to multiple apoptosis signaling pathways. Various studies have demonstrated that many components of the Akt signaling pathway are constitutively active in a wide range of human tumors, especially in leukemia ${ }^{[45]}$. Leukemia cells display increased expression of a phosphorylated form of Akt compared with granulocytes ${ }^{[46]}$. Additionally, the phosphorylation levels of GSK3 $\beta$ and S6k1, which stimulate protein synthesis and cell survival as downstream effectors of Akt, are also high in leukemia cells ${ }^{[45]}$.

It has been reported that the overexpression of SRC-3 will block apoptosis by enhancing Akt and NF-KB activity and increasing Bcl-2 expression ${ }^{[43]}$, while knockdown of SRC-3 by siRNA will decrease the expression of multiple genes associated with the Akt signal pathway and induce apoptosis in cancer cells ${ }^{[4]}$. Moreover, in the SRC-3 null mouse animal model, several components of the Akt signal pathway are also downregulated ${ }^{[47]}$. These phenomena imply that the Akt signal pathway is under the strict control of SRC-3. Consistent with previous findings, we found that Akt and its downstream targets GSK3 $\beta$ and S6k1 were constitutively activated in K562 cells, and GA inhibited their phosphorylation in a dose-dependent manner ${ }^{[44]}$, while their un-phosphorylated levels were not affected. In K562 cells, GA downregulated both the protein and mRNA levels of Bcl-2, which can act as an anti-apoptosis protein by maintaining mitochondrial membrane potential stability and is a crucial mediator downstream of the Akt signal pathway ${ }^{[48]}$.

\section{Regulation of death inducer-obliterator (DIO-1) and NF- KB}

DIO-1 was identified by differential display PCR in pre-B WOL-1 cells undergoing apoptosis by interleukin-7 (IL-7) 
starvation. The DIO-1 gene is located at 20q13.33 in Homo sapiens, and its amino acid sequence exhibits predicted transcriptional activation domains consisting of a canonical bipartite nuclear localization signal (NLS) in the $\mathrm{N}$-terminal region, two Zn-finger motifs in the central region, and a C-terminal lysine-rich sequence ${ }^{[49]}$. The expression level of DIO-1 is low in WOL-1 cells in the logarithmic growth period. When apoptosis was induced in WOL-1 cells, the DIO-1 level increased. For example, DIO-1 was upregulated in MOL-1 cells treated with IFN-ץ, dexamethasone or IL-7 deprivation but not in cells treated with etoposide or UV irradiation or cells undergoing p53-induced cell death. In MEF(10.1)Val5MycER cells, DIO-1 upregulation was observed in the absence of serum after addition of E2 but not before or at $32^{\circ} \mathrm{C}$. Transfection of a DIO-1 expression plasmid into BA/F3, A20, or FL5.12 cells resulted in cell apoptotic death, while the empty vector had no effect on cell survival; apoptotic cell death could be blocked by incubation with the pan caspase inhibitor benzyloxycarbonylVal-Ala-Asp-fluoromethyl ketone (z-VAD-fmk) or Bcl-2 overexpression $^{[49]}$.

These data implied that DIO-1 upregulation could lead to cell apoptosis by activating caspase. Garcia-Domingo et al ${ }^{[50]}$ further confirmed that nuclear translocation was the main regulatory event in the DIO-1-induced apoptotic death pathway, as DIO-1 translocation upregulated protein levels of procaspase-3 and -9, which enhanced their own apoptosis-inducing activity. A nuclear localization signal deletion mutant of DIO-1 failed to translocate to the nuclear compartment in the absence of interleukin-3, upregulate procaspase levels or trigger cell death.

In a clinical study, $100 \%$ of human myelodysplastic syndrome (MDS)/myeloproliferative disorder (MPD) patients who were analyzed showed DIO misexpression, which was also found in other myeloid neoplasm patients but not in lymphoid neoplasm patients or healthy donors ${ }^{[51]}$. In our previous study, we showed that GA could not only upregulate the DIO-1 protein expression level in a dose-dependent manner, but could also change the location of DIO-1 in adult acute lymphoblastic leukemia Jurkat T cells. In the control group, DIO-1 was mainly located in the cytoplasm, although the nucleus showed slightly scattered green fluorescence of DIO-1, and the nucleus per se was intact. After Jurkat T cells were treated with GA, DIO-1 was mainly located in the nucleus and became aggregated in each early apoptotic cell, which exhibited relatively intact but condensed chromatins ${ }^{[52]}$.

NF- $\mathrm{KB}$, as a collective term for a small family of dimeric transcription factors containing p65, RelB, c-Rel, p50/p105, and p52/p100, plays a pivotal role in lymphocyte development, proliferation, and survival and is pivotal in lymphoid malignancies, such as Hodgkin lymphoma (HL), non-Hodgkin lymphoma (NHL) and multiple myeloma (MM). When activated, NF-KB proteins trans-activate target genes encoding regulators of the cell cycle (eg, cyclin D1 and cyclin D2), antiapoptotic genes (eg, $\mathrm{Bcl}-2$ and $\mathrm{Bcl}-\mathrm{xL}$ ), angiogenesis regulators (eg, VEGF) and drug efflux pumps (MDR1) ${ }^{[53]}$. In Jurkat cells, NF-KB is highly expressed and may contribute to the overex- pression of Bcl-2, which inhibits cell apoptosis. Overexpression of Bcl-2 could also block DIO-1-induced apoptosis ${ }^{[50]}$.

In normal cells, the balance between NF-KB-regulated cell proliferation and DIO-1-mediated apoptosis may be a dynamic equilibrium. However, in some tumor cells, overexpression of NF-KB will block DIO-1-mediated apoptosis by upregulating Bcl-2 protein expression. In our experiment, GA downregulated Bcl-2 expression by inhibiting NF-kB and caused DIO-1 upregulation and a change in localization. These results contributed to pro-caspase 3 activation and induced Jurkat $\mathrm{T}$ cell apoptosis $^{[52]}$.

Consistent with the results above, we confirmed that GA could induce apoptosis in Burkitt's lymphoma Raji cells by upregulation of DIO-1 and downregulation of NF- $\mathrm{kB}$ and $\mathrm{Bcl}-\mathrm{xL}$. Together, these effects contributed to the activation of caspase-3, leading to Raji cell apoptosis ${ }^{[54]}$. Recently, LU L et $a l^{[55]}$ reported that GA could inhibit the TNF-a-induced invasion of human prostate cancer PC3 cells by inhibiting the NF-kB signaling pathway.

\section{Regulation of HERG}

Ion channels are membrane proteins that balance the transport of ions through the hydrophobic lipid bilayer of the cell membrane. Generally speaking, they play an important role in maintaining normal organism functions, such as regulation of blood pressure, nerve/muscle excitation, and sperm motility or capacitation. $\mathrm{K}^{+}$channels are the main determinants of the resting membrane potential of cells. Studies suggest that $\mathrm{K}^{+}$channels are important regulators of cell proliferation. The activation of $\mathrm{K}^{+}$channels is essential for the progression of cells through the $\mathrm{G}_{1}$ phase to the $S$ phase of the cell cycle ${ }^{[56]}$.

The human eag-related gene (herg), which encodes a channel contributing to the cardiac repolarizing current $\mathrm{I}_{\mathrm{kr}}$, belongs to an evolutionary conserved multigenic family of voltage-activated $\mathrm{K}^{+}$channels, the eag (ether a-go-go) family. Although a tiny current, $\mathrm{I}_{\mathrm{herg}}$ has profound physiological importance in cardiac rhythm ${ }^{[57]}$. Mutations of this channel cause long Q-T syndrome 2, leading to cardiac arrhythmias and sudden death. The acquisition of functional mutations in this channel contributes to short Q-T syndrome and sudden infant death ${ }^{[58]}$. Moreover, recent studies demonstrated that HERG was abundantly expressed in a variety of tumor cell lines of different histogenesis but absent in the healthy cells from which the respective tumor cells were derived ${ }^{[59]}$. HERG was not expressed in resting peripheral mononuclear cells (PBMNCs) obtained from normal donors or CD34 ${ }^{+}$cells collected from peripheral blood $\left(\mathrm{PBCD} 34^{+}\right)$. However, HERG was rapidly upregulated in the latter upon induction of proliferation of PBMNCs or PBCD34 $4^{+}$by cytokines/growth factors. This activation is necessary for CFU-GM proliferation in vitro ${ }^{[57]}$.

Various myeloid leukemia cell lines, including K562 and HL60, express herg and HERG channels. It has been reported that HERG is expressed in 36/46 (78\%) of AML patients examined, with the highest incidence in the M1 (AML without maturation), M2 (AML with maturation), M3 (acute promy- 
elocytic leukemia) and M4 (acute myelomonocytic leukemia) groups. In addition, M2 and M4 accounted for the majority of the AML patients examined ${ }^{[57]}$. Moreover, HERG channel inhibitors (antiarrhythmic drugs, such as E4031 and Way 123, 398 , or $\mathrm{CsCl}$ ) had the potential to inhibit the proliferation of FLG 29.1 and K562 cells and primary AML cells derived from AML patients and arrest them at the $\mathrm{G}_{1}$ phase of the cell cycle, while the same inhibitors were not able to block the proliferation of cell lines not expressing HERG ${ }^{[57]}$.

These studies demonstrated that the HERG channel is an important regulator of proliferation and a prerequisite for the $\mathrm{G}_{1} / \mathrm{S}$ progression of the leukemia cell lines, primary AML, and PBCD $34^{+}$cells. HERG was also upregulated in lymphoid leukemia. The HERG transcript level was not correlated with the B-cell subset, as it was elevated in both immature neoplastic B-CLL cells $\left(\mathrm{CD}^{+}\right)$and a CD5 Burkitt's lymphoma Raji cell line. However, in Sjögren's syndrome cells (enriched in $\mathrm{CD}^{+}$ B-cells) or Epstein-Barr virus-transformed B-cells (CD5 cells), the HERG expression level was not elevated ${ }^{[60]}$. Pillozzi et al ${ }^{[61]}$ further revealed that a HERG $\mathrm{K}^{+}$channel, FLT- 1 and $\beta_{1}$ integrin could form a macromolecular signaling complex, which mostly recruited the HERG1B isoform of the HERG channel, and its assembly was necessary for AML cell migration. The complex was also found in primary AML blasts obtained from AML patients. The co-expression of FLT-1 and HERG conferred a pro-migratory phenotype to AML blasts. The HERGpositive blasts were more likely to invade the peripheral circulation and extramedullary sites after engraftment into NOD-SCID mice. Furthermore, HERG expression was associated with a higher probability of relapse and shorter survival periods in leukemia patients. These data demonstrated that the HERG channel not only promoted the proliferation of leukemia cells but also conferred a greater abilityto migrate and invade ${ }^{[61]}$.

In addition to leukemia cells, the HERG gene and HERG protein are also expressed in many colon cancer cells, such as the DLD1, HCT8, HCT116, and H630 cell lines. In particular, HCT116 and H630 cells expressed HERG at the highest level, whereas HCT8 cells expressed it at the lowest level. The activity of the HERG channel regulated the cell invasiveness of these colon cancer cell lines, and the amount of HERG protein was correlated with a more invasive phenotype of colon cancer cells. Although no expression of the HERG channel has been detected in normal human colonic mucosa or adenomas, a high percentage of primary colorectal cancers express the HERG channel, with a higher incidence in metastatic cancers $^{[62]}$, which is consistent with the hypothesis above that high expression of the HERG channel contributed to the invasion and migration of leukemia cells.

From the above studies, it is clear that blocking the HERG channel will inhibit tumor cell proliferation, invasion and migration and thus block tumor disease progression and prolong the survival time of cancer patients. For example, knocking down HERG gene expression using short hairpin RNA (shRNA) for the HERG1 and HERG1b isoforms reduced the growth rate and cell viability of neuroblastoma cells, inhibited their colony formation, and restricted them to the $G_{0} / G_{1}$ phase of the cell cycle. Moreover, treatment with shRNA for HERG also inhibited tumor cell growth when injected into nude mice ${ }^{[63]}$.

In our previous study, we first revealed that GA had the potential ability to downregulate HERG mRNA and protein expression in chronic myeloid leukemia K562 cells in a dosedependent manner. Consistent with the viewpoint that the HERG channel is an important modulator of the progression of cells through $\mathrm{G}_{1}$ phase to $\mathrm{S}$ phase of the cell cycle ${ }^{[56]}, \mathrm{K} 562$ cells treated with $G A$ were arrested at $\mathrm{G}_{0} / \mathrm{G}_{1}$ phase, while the rate of $S$ phase cells decreased, which was accompanied by the downregulation of the HERG channel. As the concentration of GA increased, the expression of HERG protein in K562 cells decreased, while the rate of cells in $G_{0} / G_{1}$ phase increases. These studies implied that GA could arrest K562 cells at the $\mathrm{G}_{0} / \mathrm{G}_{1}$ phase of the cell cycle, perhaps by blocking HERG channel conformation in the tumor cells ${ }^{[64]}$.

\section{Induction of reactive oxygen species (ROS) and their accumulation}

ROS, a term that describes the superoxide anion, hydroxyl, peroxyl, alkoxyl and $\mathrm{O}_{2}$-derived non-radical species, such as hydrogen peroxide, are generally highly reactive and shortlived $^{[65}$. ROS are mainly produced by mitochondria through electron leakage form complexes I and III. ROS are also produced by NAD $(\mathrm{P}) \mathrm{H}$ oxidases.

Lastly, some metabolic enzymes often create ROS through nonspecific reactions ${ }^{[66]}$. As products or by-products of the process of cell metabolism, ROS can act as either signaling molecules or cell toxicants depending on their generated site, spatial distribution, pulse concentration and temporal duration ${ }^{[67]}$. ROS levels are mainly regulated by non-enzymatic antioxidants (eg, glutathione) and antioxidant enzymes (eg, superoxide dismutase $)^{[68]}$. If the balance between ROS levels and antioxidants tips toward the oxidant side, ROS will accumulate, which leads to damage to many biomolecules, such as DNA, protein and lipids ${ }^{[69]}$. It has been reported that disorders of ROS are associated with some diseases, including Alzheimer's disease and Parkinson's disease ${ }^{[70]}$. However, ROS also contribute to the anticancer activity of some chemotherapy ${ }^{[71]}$.

It has been reported that ROS can activate caspases, which induce apoptosis by cleaving substrates, such as cell cycleand DNA repair-related proteins, and the mediators of apoptosis $^{[72]}$. ROS can induce the collapse of the mitochondrial membrane potential (MMP), leading to the release of factor cytochrome $c$ from the inner mitochondrial membrane into the cytosol, which activates the apoptosis executioner caspase-3 through activation of the apoptosis initiator caspase- $9^{[73]}$. Moreover, the release of cytochrome $c$ enhances the accumulation of $\operatorname{ROS}^{[74]}$.

The structure of GA includes an $\alpha, \beta$-unsaturated ketone, and structure-activity relationship analysis implied that the $\alpha, \beta$-unsaturated ketone present in GA is relevant to its cytotoxicity ${ }^{[75]}$. As some drugs that contain unsaturated ketones were able to induce apoptosis via the accumulation of 
$\operatorname{ROS}^{[76,77]}$, we presumed that GA could also induce apoptosis in multiple myeloma RPMI8226 cells via ROS generation.

In our study, we found that GA induced RPMI8226 cell apoptosis and inhibited proliferation in a dose-dependent manner, and these changes were accompanied by the activation of caspse-3 and cleavage of PARP-1. At the same time, the concentration of ROS was also deregulated in RPMI8226 cells treated with GA in a dose-dependent manner. To verify the hypothesis that ROS accumulation contributes to the apoptosis caused by GA, the ROS scavenger N-acetylcysteine (NAC) was used. Strikingly, NAC significantly blocked the ability of GA to induce RPMI8226 cell apoptosis and alleviated the accumulation of ROS. The activation of caspase- 3 and the cleavage of PARP1 caused by GA were also inhibited by the addition of NAC. This result was consistent with reports that GA could induce apoptosis in human hepatoma SMMC-7721 cells via ROS accumulation ${ }^{[5]}$.

\section{Conclusions}

The natural product GA is a promising novel antitumor agent that acts via various mechanisms in solid tumors and hematological malignancies. GA can be exploited in different malignancies that are refractory to standard care because it acts through numerous antitumor mechanisms. GA has the potential to selectively kill neoplastic cells but spare normal cells ${ }^{[4]}$ and is currently in clinical trials in China. In a phase I human tolerability trial of GA injection, Wang et al reported that the maximal tolerated dose (MTD) of a single injection was 55 $\mathrm{mg} / \mathrm{m}^{2}$, and the main dose-limiting toxicities (DLTs) were liver dysfunction and pain ${ }^{[78]}$. Another phase II clinical trial, in which all 50 cases were advanced solid tumor patients with tumors that were not sensitive to conventional chemotherapy, was conducted. The patients were treated with $45 \mathrm{mg} / \mathrm{m}^{2}$ GA (qd or qod) five times in a 14-d cycle. After two cycles, 47 cases were eligible for final analysis, and the responses were PR 3, SD 29, and PD 15 $5^{[79]}$. As GA has the potential to induce apoptosis in various cell lines derived from respective hematological malignancies, we believe that GA can also be exploited for curing patients with various hematological malignances.

\section{Acknowledgements}

This work was supported by the National Natural Science Foundation of China (81070429).

\section{References}

1 Broker LE, Kruyt FA, Giaccone G. Cell death independent of caspases: a review. Clin Cancer Res 2005; 11: 3155-62.

2 Fulda S. Betulinic acid: a natural product with anticancer activity. Mol Nutr Food Res 2009; 53: 140-6.

3 Hanahan D, Weinberg RA. Hallmarks of cancer: the next generation. Cell 2011; 144: 646-74.

4 Prasad S, Pandey MK, Yadav VR, Aggarwal BB. Gambogic acid inhibits STAT3 phosphorylation through activation of protein tyrosine phosphatase SHP-1: potential role in proliferation and apoptosis. Cancer Prev Res (Phila) 2011; 4: 1084-94.

5 Nie F, Zhang X, Qi Q, Yang L, Yang Y, Liu W, et al. Reactive oxygen species accumulation contributes to gambogic acid-induced apoptosis in human hepatoma SMMC-7721 cells. Toxicology 2009; 260: 60-7.

6 Wu ZQ, Guo QL, You QD, Zhao L, Gu HY. Gambogic acid inhibits proliferation of human lung carcinoma SPC-A1 cells in vivo and in vitro and represses telomerase activity and telomerase reverse transcriptase mRNA expression in the cells. Biol Pharm Bull 2004; 27: 1769-74.

7 Guo QL, You QD, Wu ZQ, Yuan ST, Zhao L. General gambogic acids inhibited growth of human hepatoma SMMC-7721 cells in vitro and in nude mice. Acta Pharmacol Sin 2004; 25: 769-74.

8 Xie H, Qin YX, Zhou YL, Tong LJ, Lin LP, Geng MY, et al. GA3, a new gambogic acid derivative, exhibits potent antitumor activities in vitro via apoptosis-involved mechanisms. Acta Pharmacol Sin 2009; 30: 346-54.

9 Yang Y, Yang L, You QD, Nie FF, Gu HY, Zhao L, et al. Differential apoptotic induction of gambogic acid, a novel anticancer natural product, on hepatoma cells and normal hepatocytes. Cancer Lett 2007; 256: 259-66.

10 Kau TR, Way JC, Silver PA. Nuclear transport and cancer: from mechanism to intervention. Nat Rev Cancer 2004; 4: 106-17.

11 Jamali T, Jamali Y, Mehrbod M, Mofrad MR. Nuclear pore complex: biochemistry and biophysics of nucleocytoplasmic transport in health and disease. Int Rev Cell Mol Biol 2011; 287: 233-86.

12 Xu S, Powers MA. Nuclear pore proteins and cancer. Semin Cell Dev Biol 2009; 20: 620-30.

13 Hashizume C, Nakano H, Yoshida K, Wong RW. Characterization of the role of the tumor marker Nup88 in mitosis. Mol Cancer 2010; 9: 119.

14 Fornerod M, van Deursen J, van Baal S, Reynolds A, Davis D, Murti $K G$, et al. The human homologue of yeast CRM1 is in a dynamic subcomplex with CAN/Nup214 and a novel nuclear pore component Nup88. EMBO J 1997; 16: 807-16.

15 Roth P, Xylourgidis N, Sabri N, Uv A, Fornerod M, Samakovlis C. The Drosophila nucleoporin DNup88 localizes DNup214 and CRM1 on the nuclear envelope and attenuates NES-mediated nuclear export. J Cell Biol 2003; 163: 701-6.

16 Xylourgidis N, Roth P, Sabri N, Tsarouhas V, Samakovlis C. The nucleoporin Nup214 sequesters CRM1 at the nuclear rim and modulates NFkappaB activation in Drosophila. J Cell Sci 2006; 119: 4409-19.

17 Schneider J, Moragues D, Martinez N, Romero H, Jimenez E, Ponton J. Cross-reactivity between Candida albicans and human ovarian carcinoma as revealed by monoclonal antibodies PA10F and C6. Br J Cancer 1998; 77: 1015-20.

18 Gould VE, Orucevic A, Zentgraf H, Gattuso P, Martinez N, Alonso A. Nup88 (karyoporin) in human malignant neoplasms and dysplasias: correlations of immunostaining of tissue sections, cytologic smears, and immunoblot analysis. Hum Pathol 2002; 33: 536-44.

19 Agudo D, Gomez-Esquer F, Martinez-Arribas F, Nunez-Villar MJ, Pollan $\mathrm{M}$, Schneider J. Nup88 mRNA overexpression is associated with high aggressiveness of breast cancer. Int J Cancer 2004; 109: 717-20.

20 Takahashi N, van Kilsdonk JW, Ostendorf B, Smeets R, Bruggeman $\mathrm{SW}$, Alonso A, et al. Tumor marker nucleoporin $88 \mathrm{kDa}$ regulates nucleocytoplasmic transport of NF-kappaB. Biochem Biophys Res Commun 2008; 374: 424-30.

21 Shu W, Chen Y, He J, Cui G. Effects of gambogic acid on the regulation of nucleoporin Nup88 in U937 cells. J Huazhong Univ Sci Technolog Med Sci 2007; 27: 388-92.

22 Shu WX, Chen Y, He J. Effects of gambogic acid on the regulation of nucleoporin Nup88 in HL-60 cells. Zhonghua Zhong Liu Za Zhi 2008; 30: 484-9.

23 Shu W, Chen Y, Li R, Wu Q, Cui G, Ke W, et al. Involvement of regula- 
tions of nucleophosmin and nucleoporins in gambogic acid-induced apoptosis in Jurkat cells. Basic Clin Pharmacol Toxicol 2008; 103: 530-7.

24 Yun JP, Chew EC, Liew CT, Chan JY, Jin ML, Ding MX, et al. Nucleophosmin/B23 is a proliferate shuttle protein associated with nuclear matrix. J Cell Biochem 2003; 90: 1140-8.

25 Grisendi S, Mecucci C, Falini B, Pandolfi PP. Nucleophosmin and cancer. Nat Rev Cancer 2006; 6: 493-505.

26 Falini B, Mecucci C, Tiacci E, Alcalay M, Rosati R, Pasqualucci L, et al. Cytoplasmic nucleophosmin in acute myelogenous leukemia with a normal karyotype. N Engl J Med 2005; 352: 254-66.

$27 \mathrm{Hsu} \mathrm{CY}$, Yung BY. Involvement of nucleophosmin/B23 in TPA-induced megakaryocytic differentiation of K562 cells. Br J Cancer 2003; 89: 1320-6.

28 Hsu CY, Yung BY. Over-expression of nucleophosmin/B23 decreases the susceptibility of human leukemia HL-60 cells to retinoic acidinduced differentiation and apoptosis. Int J Cancer 2000; 88: 392400.

29 Suen CS, Berrodin TJ, Mastroeni R, Cheskis BJ, Lyttle CR, Frail DE. A transcriptional coactivator, steroid receptor coactivator-3, selectively augments steroid receptor transcriptional activity. J Biol Chem 1998; 273: 27645-53.

30 Gojis O, Rudraraju B, Alifrangis C, Krell J, Libalova P, Palmieri C. The role of steroid receptor coactivator-3 (SRC-3) in human malignant disease. Eur J Surg Oncol 2010; 36: 224-9.

$31 \mathrm{Ma} \mathrm{G}$, Ren Y, Wang K, He J. SRC-3 has a role in cancer other than as a nuclear receptor coactivator. Int J Biol Sci 2011; 7: 664-72.

32 Amazit L, Pasini L, Szafran AT, Berno V, Wu RC, Mielke M, et al. Regulation of SRC-3 intercompartmental dynamics by estrogen receptor and phosphorylation. Mol Cell Biol 2007; 27: 6913-32.

33 Anzick SL, Kononen J, Walker RL, Azorsa DO, Tanner MM, Guan XY, et al. AIB1, a steroid receptor coactivator amplified in breast and ovarian cancer. Science 1997; 277: 965-8.

34 Tanner MM, Grenman S, Koul A, Johannsson O, Meltzer P, Pejovic T, et al. Frequent amplification of chromosomal region 20q12-q13 in ovarian cancer. Clin Cancer Res 2000; 6: 1833-9.

35 Glaeser M, Floetotto T, Hanstein B, Beckmann MW, Niederacher D. Gene amplification and expression of the steroid receptor coactivator SRC3 (AIB1) in sporadic breast and endometrial carcinomas. Horm Metab Res 2001; 33: 121-6.

36 He LR, Liu MZ, Li BK, Rao HL, Deng HX, Guan XY, et al. Overexpression of AIB1 predicts resistance to chemoradiotherapy and poor prognosis in patients with primary esophageal squamous cell carcinoma. Cancer Sci 2009; 100: 1591-6.

37 Grivas PD, Tzelepi V, Sotiropoulou-Bonikou G, Kefalopoulou Z, Papavassiliou AG, Kalofonos H. Estrogen receptor alpha/beta, AIB1, and TIF2 in colorectal carcinogenesis: do coregulators have prognostic significance? Int J Colorectal Dis 2009; 24: 613-22.

38 Xu Y, Chen Q, Li W, Su X, Chen T, Liu Y, et al. Overexpression of transcriptional coactivator AIB1 promotes hepatocellular carcinoma progression by enhancing cell proliferation and invasiveness. Oncogene 2010; 29: 3386-97.

39 Henke RT, Haddad BR, Kim SE, Rone JD, Mani A, Jessup JM, et al. Overexpression of the nuclear receptor coactivator AIB1 (SRC-3) during progression of pancreatic adenocarcinoma. Clin Cancer Res 2004; 10: 6134-42.

40 Torres-Arzayus MI, Font de Mora J, Yuan J, Vazquez F, Bronson R, Rue M, et al. High tumor incidence and activation of the PI3K/AKT pathway in transgenic mice define AIB1 as an oncogene. Cancer Cell 2004; 6: 263-74.

41 Colo GP, Rosato RR, Grant S, Costas MA. RAC3 down-regulation sensitizes human chronic myeloid leukemia cells to TRAlL-induced apoptosis. FEBS Lett 2007; 581: 5075-81.

42 Esteyries S, Perot C, Adelaide J, Imbert M, Lagarde A, Pautas C, et al. NCOA3, a new fusion partner for MOZ/MYST3 in M5 acute myeloid leukemia. Leukemia 2008; 22: 663-5.

43 Colo GP, Rubio MF, Nojek IM, Werbajh SE, Echeverria PC, Alvarado CV, et al. The p160 nuclear receptor co-activator RAC3 exerts an antiapoptotic role through a cytoplasmatic action. Oncogene 2008; 27 : 2430-44.

44 Li R, Chen Y, Zeng LL, Shu WX, Zhao F, Wen L, et al. Gambogic acid induces $G_{0} / G_{1}$ arrest and apoptosis involving inhibition of SRC-3 and inactivation of Akt pathway in K562 leukemia cells. Toxicology 2009; 262: 98-105.

45 Wang Z, Smith KS, Murphy M, Piloto O, Somervaille TC, Cleary ML. Glycogen synthase kinase 3 in MLL leukaemia maintenance and targeted therapy. Nature 2008; 455: 1205-9.

46 Tazzari PL, Cappellini A, Grafone T, Mantovani I, Ricci F, Billi AM, et al. Detection of serine 473 phosphorylated Akt in acute myeloid leukaemia blasts by flow cytometry. Br J Haematol 2004; 126: 67581.

47 Yan J, Yu CT, Ozen M, Ittmann M, Tsai SY, Tsai MJ. Steroid receptor coactivator-3 and activator protein-1 coordinately regulate the transcription of components of the insulin-like growth factor/AKT signaling pathway. Cancer Res 2006; 66: 11039-46.

48 Asnaghi L, Calastretti A, Bevilacqua A, D'Agnano I, Gatti G, Canti G, et al. Bcl-2 phosphorylation and apoptosis activated by damaged microtubules require $\mathrm{mTOR}$ and are regulated by Akt. Oncogene 2004; 23: 5781-91.

49 Garcia-Domingo D, Leonardo E, Grandien A, Martinez P, Albar JP, Izpisua-Belmonte $\mathrm{JC}$, et al. DIO-1 is a gene involved in onset of apoptosis in vitro, whose misexpression disrupts limb development. Proc Natl Acad Sci U S A 1999; 96: 7992-7.

50 Garcia-Domingo D, Ramirez D, Gonzalez de Buitrago G, Martinez AC. Death inducer-obliterator 1 triggers apoptosis after nuclear translocation and caspase upregulation. Mol Cell Biol 2003; 23: 3216-25.

51 Futterer A, Campanero MR, Leonardo E, Criado LM, Flores JM, Hernandez JM, et al. Dido gene expression alterations are implicated in the induction of hematological myeloid neoplasms. J Clin Invest 2005; 115: 2351-62.

52 Wang Y, Chen Y, Chen Z, Wu Q, Ke WJ, Wu QL. Gambogic acid induces death inducer-obliterator 1-mediated apoptosis in Jurkat T cells. Acta Pharmacol Sin 2008; 29: 349-54.

53 Packham G. The role of NF-kappaB in lymphoid malignancies. $\mathrm{Br} J$ Haematol 2008; 143: 3-15.

54 Wang Y, Chen Y, Chen Z, Ke WJ, Wu QL, He J. Mechanism of gambogic acid-induced apoptosis in Raji cells. Zhongguo Shi Yan Xue Ye Xue Za Zhi 2009; 17: 88-91.

55 Lu L, Tang D, Wang L, Huang LQ, Jiang GS, Xiao XY, et al. Gambogic acid inhibits TNF-alpha-induced invasion of human prostate cancer PC3 cells in vitro through PI3K/Akt and NF-kappaB signaling pathways. Acta Pharmacol Sin 2012; 33: 531-41.

56 Wonderlin WF, Strobl JS. Potassium channels, proliferation and $\mathrm{G}_{1}$ progression. J Membr Biol 1996; 154: 91-107.

57 Pillozzi S, Brizzi MF, Balzi M, Crociani O, Cherubini A, Guasti L, et al. HERG potassium channels are constitutively expressed in primary human acute myeloid leukemias and regulate cell proliferation of normal and leukemic hemopoietic progenitors. Leukemia 2002; 16 : 1791-8.

58 Asher V, Sowter H, Shaw R, Bali A, Khan R. Eag and HERG potassium channels as novel therapeutic targets in cancer. World J Surg Oncol 
2010; 8: 113.

59 Wang $\mathrm{H}$, Zhang $\mathrm{Y}$, Cao L, Han H, Wang J, Yang B, et al. HERG K $\mathrm{K}^{+}$ channel, a regulator of tumor cell apoptosis and proliferation. Cancer Res 2002; 62: 4843-8.

60 Smith GA, Tsui HW, Newell EW, Jiang X, Zhu XP, Tsui FW, et al. Functional up-regulation of HERG $\mathrm{K}^{+}$channels in neoplastic hematopoietic cells. J Biol Chem 2002; 277: 18528-34.

61 Pillozzi S, Brizzi MF, Bernabei PA, Bartolozzi B, Caporale R, Basile V, et al. VEGFR-1 (FLT-1), beta1 integrin, and hERG $\mathrm{K}^{+}$channel for a macromolecular signaling complex in acute myeloid leukemia: role in cell migration and clinical outcome. Blood 2007; 110: 1238-50.

62 Lastraioli E, Guasti L, Crociani O, Polvani S, Hofmann G, Witchel H, et al. herg1 gene and HERG1 protein are overexpressed in colorectal cancers and regulate cell invasion of tumor cells. Cancer Res 2004; 64: 606-11.

63 Zhao J, Wei XL, Jia YS, Zheng JQ. Silencing of herg gene by shRNA inhibits SH-SY5Y cell growth in vitro and in vivo. Eur J Pharmacol 2008; 579: 50-7.

64 Cui G, Shu W, Wu Q, Chen Y. Effect of Gambogic acid on the regulation of hERG channel in K562 cells in vitro. J Huazhong Univ Sci Technolog Med Sci 2009; 29: 540-5.

65 Scherz-Shouval R, Elazar Z. Regulation of autophagy by ROS: physiology and pathology. Trends Biochem Sci 2011; 36: 30-8.

66 Morgan MJ, Liu ZG. Crosstalk of reactive oxygen species and NFkappaB signaling. Cell Res 2011; 21: 103-15.

67 Liu B, Chen Y, St Clair DK. ROS and p53: a versatile partnership. Free Radic Biol Med 2008; 44: 1529-35.

68 Terada LS. Specificity in reactive oxidant signaling: think globally, act locally. J Cell Biol 2006; 174: 615-23.

69 Jones DP. Redefining oxidative stress. Antioxid Redox Signal 2006; 8: 1865-79.

70 Valko M, Leibfritz D, Moncol J, Cronin MT, Mazur M, Telser J. Free radicals and antioxidants in normal physiological functions and human disease. Int J Biochem Cell Biol 2007; 39: 44-84.

71 Chen Y, Jungsuwadee P, Vore M, Butterfield DA, St Clair DK. Collateral damage in cancer chemotherapy: oxidative stress in nontargeted tissues. Mol Interv 2007; 7: 147-56.

72 Marnett $\sqcup$, Riggins JN, West JD. Endogenous generation of reactive oxidants and electrophiles and their reactions with DNA and protein. J Clin Invest 2003; 111: 583-93.

73 Circu ML, Aw TY. Reactive oxygen species, cellular redox systems, and apoptosis. Free Radic Biol Med 2010; 48: 749-62.

74 Slee EA, Harte MT, Kluck RM, Wolf BB, Casiano CA, Newmeyer DD, et al. Ordering the cytochrome $c$-initiated caspase cascade: hierarchical activation of caspases-2, -3, -6, -7, -8, and -10 in a caspase-9dependent manner. J Cell Biol 1999; 144: 281-92.

75 Kasibhatla S, Jessen KA, Maliartchouk S, Wang JY, English NM, Drewe $\mathrm{J}$, et al. A role for transferrin receptor in triggering apoptosis when targeted with gambogic acid. Proc Natl Acad Sci U S A 2005; 102 : 12095-100.

76 Chen YC, Shen SC, Tsai SH. Prostaglandin $D_{2}$ and $J_{2}$ induce apoptosis in human leukemia cells via activation of the caspase 3 cascade and production of reactive oxygen species. Biochim Biophys Acta 2005; 1743: 291-304.

77 Kondo M, Shibata T, Kumagai T, Osawa T, Shibata N, Kobayashi M, et al. 15-Deoxy-Delta(12,14)-prostaglandin J(2): the endogenous electrophile that induces neuronal apoptosis. Proc Natl Acad Sci U S A 2002; 99: 7367-72.

78 Zhou Z, Wang J. Phase I human tolerability trial of gambogic acid. Chin J New Drugs 2007; 16: 79-83.

79 Zhan $X$. The summary report of Phase Ila clinical trial of gambogic acid injection [dissertation]. Peking: Peking Union Medical College; 2008. 